\section{POLÍTICAS SOBRE INNOVACIÓN EDUCATIVA EN CLAVE AUTONÓMICA. LA COMUNIDAD DE MADRID}

\author{
Xavier Gisbert \\ Director General de mejora de la Calidad \\ de la Enseñanza de la Comunidad de Madrid
}

\section{POLICIES ON EDUCATIONAL INNOVATION IN AUTONOMOUS KEY. COMUNIDAD DE MADRID}

\begin{abstract}
The importance of the educational innovation has been enforced by the decision taken by the European Parliament in order to declare that 2009 is the "European Year for Creativity and Innovation". The "Consejería de Educación de la CAM" (Madrid Autonomous Community Education Committee) has introduced the innovation in the teachers' day-to-day life. Bilingual schools, linguistic sections, the promotion of the educational use of information and communication technologies and initiatives such as the General Plan for the improvement of the skills, the plan for the promotion of the reading, the school championships, the teachers' education and training and the publications which have meant and mean a great step forward in the Educational Innovation in the CAM.
\end{abstract}

KEY WORDS: Improvement of the education system; innovation; bilingual schools; innovation and communication technologies; outof-school activities; school championships; teachers' education and training; publications; educational innovation in the CAM (Madrid's Autonomous Community).

\section{INNOVACIÓN Y EDUCACIÓN}

La Real Academia Española define innovación como la acción y efecto de innovar, es decir mudar o alterar algo, introduciendo novedades y como la creación o modificación de un producto, y su introducción en el mercado. Innovar también significa para muchos romper moldes. Las leyes educativas, en especial las dos últimas, ya que la Ley Orgánica de Eduación (LOE) reproduce en su totalidad a la derogada Ley de Calidad de la Enseñanza (LOCE) en lo que a innovación se refiere, hacen referencia a la innovación en el sistema educativo vinculándola a sus principios generales, a la formación permanente del profesorado, a la mejora profesional del profesorado y a la dirección de los centros. En realidad traslada a las administraciones educativas la competencia de desarrollar y fomentar políticas de innovación en sus centros. En este marco cada Consejería
RESUMEN: La importancia de la innovación educativa se ha visto reforzada por la decisión del Parlamento Europeo de declarar 2009 "Año Europeo de la Creatividad y la Innovación". La Consejería de Educación de la CAM ha introducido la innovación en el dia a dia de los profesores. Colegios bilingües, secciones lingüisticas, fomento del uso educativo de las Tecnologías de la Información y la Comunicación, iniciativas como El Plan General de Mejora de las Destrezas y El Plan de Fomento de la Lectura, los Campeonatos Escolares, la Formación del Profesorado y las Publicaciones han supuesto y suponen un gran avance de la Innovación Educativa en la CAM.

PALABRAS CLAVE: Mejora del sistema educativo; innovación; colegios bilingües; tecnologías de la innovación y la comunicación; actividades extraescolares; campeonatos escolares; formación del profesorado; publicaciones; innovación educativa en la CAM.

de Educación debe definir sus objetivos y fijar las políticas educativas correspondientes que permitan alcanzarlos.

El sistema educativo plantea unos retos muy importantes en la actual sociedad, especialmente en un país cuyo principal bagaje es su capital humano. Necesitamos un sistema educativo que forme a nuestros jóvenes para que puedan competir en el mundo globalizado en el que nos ha tocado vivir al menos en igualdad de condiciones con el resto de jóvenes de la Unión Europea. Es evidente que nuestro actual sistema educativo no reúne los requisitos necesarios para ello, como demuestran los resultados y las evaluaciones tanto nacionales como internacionales y por lo tanto es preciso actuar desarrollando políticas que minimicen los efectos negativos de la ley. Una de las principales vías para "escapar" del corsé legal que atenaza a las administraciones educativas es la innovación, entendida 
ésta en su sentido más amplio, es decir en el desarrollo de actuaciones que tengan como objetivo la mejora del sistema educativo, y evidentemente dentro de los límites legales establecidos.

\section{AÑo EuRopeo de la creatividad y La INNOVACIÓN}

La importancia de la innovación educativa se ha visto recientemente reforzada por una decisión del Parlamento Europeo y del Consejo de declarar el año 2009 "Año Europeo de la Creatividad y la Innovación" con el objetivo de respaldar los esfuerzos de los Estados miembros para promover la creatividad y la capacidad de innovación mediante la creación de entornos favorables a la innovación, la flexibilidad y la adaptabilidad en un mundo que cambia con rapidez, en los que debe tenerse en cuenta todas las formas de innovación. Propone esta decisión el aumento de la sensibilización sobre la importancia de la creatividad, la innovación y el espíritu emprendedor tanto para el desarrollo personal como para el crecimiento económico y el empleo, así como el fomento de la mentalidad empresarial, especialmente entre los jóvenes; el fomento de la educación de las capacidades matemáticas, científicas y tecnológicas básicas y avanzadas favorables a la innovación tecnológica; el impulso de la apertura al cambio, la creatividad y la resolución de problemas como competencias favorables a la innovación y transferibles a diversos contextos profesionales y sociales; en definitiva se propone desarrollar al máximo la creatividad y la capacidad de innovación.

Para la Comunidad de Madrid, la innovación es un excelente instrumento de mejora que permite introducir, en un sistema educativo ineficaz, elementos que tienden a corregir los malos resultados a los que, de no hacerlo, nos veríamos abocados. Para ello la innovación no se contempla como actuaciones aisladas, sino como algo que permea todo el sistema y a todos los niveles. No se trata pues de limitarse a justificar la posibilidad que ofrece la ley mediante la puesta en marcha de alguna iniciativa sino más bien de conseguir que la idea, el concepto de innovación esté presente en el mayor número de ámbitos del sistema, que forme parte de él tanto de forma explícita como implícita y por lo tanto que afecte a todos los miembros del sistema educativo formando parte de su desarrollo.

\section{INNOVACIÓN Y SISTEMA EDUCATIVO}

La Consejería de Educación de la Comunidad de Madrid se caracteriza precisamente por haber sabido introducir la innovación en el día a día de profesores, alumnos y padres de manera que todos ellos cuentan con puertas abiertas y con posibilidades que les permiten mejorar sus expectativas. En un texto como el presente que no tiene por objeto ser analítico sino más bien descriptivo, la mejor manera de mostrar el carácter innovador del sistema educativo de la Comunidad de Madrid es el de presentar las actuaciones más relevantes vinculadas a ese objetivo. Se exponen por lo tanto las actuaciones y los programas más representativos y que confieren a la Consejería de Educación ese dinamismo en el desarrollo de sus políticas educativas. Entre otros el Programa de colegios bilingües, la introducción y extensión del uso de las tecnologías de la información y la comunicación en los centros mediante la dotación progresiva de pizarras digitales interactivas y sistemas de conexión, el fomento de las actividades extraescolares de carácter innovador, el desarrollo y potenciación del portal educativo, las publicaciones relacionadas con la innovación, el establecimiento de una nueva red de formación del profesorado a través de centros de innovación y formación, la realización de pruebas de conocimientos y destrezas indispensables a los alumnos de sexto de primaria y de tercero de secundaria, en fin, una bateria de actuaciones que convierte al sistema educativo de la Comunidad de Madrid, con los límites mencionados anteriormente, en un sistema innovador.

\section{Programa de colegios bilingües}

Se trata sin duda del programa estrella de la Consejería de Educación precisamente por su carácter innovador. En el curso 2004/05 la Consejería de Educación de la Comunidad de Madrid puso en marcha el conocido Programa de colegios bilingües con el fin de mejorar los niveles de los jóvenes madrileños en una materia tan importante como la lengua inglesa y ofrecerles el mayor nivel de preparación que les asegure las mejores oportunidades ante un mundo laboral y profesional cada vez más globalizado.

Las características del programa son bien conocidas y la búsqueda de la excelencia se sustenta en unos principios 
basados en la mejora de la enseñanza pública y en la igualdad de oportunidades para todos los alumnos, independientemente de diferencias de origen social, económico, o de otro tipo.

Se trata de un programa ambicioso, que se ha centrado hasta el pasado curso exclusivamente en centros públicos de la Comunidad de Madrid, de implantación progresiva y que tiene en cuenta la distribución geográfica y territorial de los centros, respetando siempre la libre voluntad de los colegios para incorporarse al mismo. Es un programa con un diseño y un desarrollo que contempla todos y cada uno de los aspectos que deben incidir en el mismo para hacerlo consistente y potente.

Su implantación y desarrollo anual se asegura mediante el establecimiento de requisitos y de procedimientos para los centros que aspiran a convertirse en bilingües. Una vez seleccionados, y en función de la preparación y la competencia lingüística de los maestros que van a participar, se ofrece a todos ellos una formación de entrada específica de un curso de cuatro semanas diseñado para aquellos maestros que precisan de un mayor apoyo y refuerzo. Todos los maestros candidatos participan en un curso de formación en una Universidad extranjera de un mes de duración. Una vez iniciado el programa, todos los maestros tienen a su disposición una variada oferta de formación permanente dentro de la red de formación de la Consejería de Educación. La formación, tanto la de entrada como la permanente constituye un elemento fundamental del programa y se cuenta para ello con acuerdos con instituciones como el British Council y con convenios con 14 universidades extranjeras de gran prestigio a través de las cuales ya han recibido formación cerca de 1.200 maestros.

Otro de los elementos que caracteriza este programa es sin duda la presencia de auxiliares de conversación, jóvenes nativos que realizan una labor fundamental de apoyo al profesorado ya que mediante el desarrollo de actividades de lengua y cultura vienen a reforzar el trabajo de nuestros especialistas. Su número se incrementa año a año siendo la media de tres auxiliares de conversación por centro en estos momentos. Los centros seleccionados así como los maestros que participan en el programa reciben unos incentivos económicos que vienen a compensar la dedicación al mismo.
La innovación se suele vincular a una proyección temporal limitada sin embargo en el caso que nos ocupa, una vez implantado, el proyecto mantiene su valor innovador ya que su implantación progresiva extiende este carácter cada curso escolar en cada uno de los centros. El programa de colegios bilingües es a la vez pionero e innovador, con un horizonte temporal inusualmente extenso que en estos momentos se halla en su quinto año de implantación y ha supuesto hasta la fecha una inversión superior a los treinta millones de euros.

El objetivo es que los alumnos adquieran a lo largo de su escolaridad, un conocimiento y uso adecuado de las dos lenguas, española e inglesa, de forma oral y escrita. Se trata en definitiva, de que sean capaces de desenvolverse con naturalidad en situaciones cotidianas y de que adquieran para ello la competencia comunicativa necesaria y son ya más de 30.000 los alumnos que reciben enseñanza bilingüe en este programa.

Nada debe ser dejado al azar y para ello se cuidan aspectos como el hermanamiento de esos centros con escuelas gemelas en el extranjero, especialmente el Reino Unido o algo tan fundamental como la evaluación del conocimiento de nuestros alumnos, que permite valorar de un modo positivo el balance de estos cinco años por medio de una prueba externa homologada internacionalmente llevada a cabo cada año por la institución Trinity College a los alumnos de $2 .^{\circ}$ y $4 .{ }^{\circ}$ de primaria, reflejando unos resultados de éxito superiores al $90 \%$.

\section{Secciones lingüísticas}

La preocupación por el bilingüismo en la Comunidad de Madrid no es un fenómeno reciente sino que tiene su origen en la puesta en marcha por la actual Presidenta de nuestra región del programa del Ministerio de Educación en colaboración con el British Council al inicio de su etapa como Ministra de Educación y Cultura en 1996. Ese programa, que se extendió a todas las provincias cuyas comunidades pertenecian al ámbito de gestión del Ministerio, ha constituido el germen de muchos programas bilingües.

Como complemento al desarrollo de programas en los niveles de educación primaria, muchas comunidades autónomas, 
y Madrid entre ellas, pusieron en marcha secciones lingüisticas en centros de secundaria. En estos momentos Madrid cuenta con 10 secciones lingüisticas de inglés, 15 de francés y 3 de alemán en Institutos de Enseñanza Secundaria con cerca de 4.000 alumnos recibiendo estas enseñanzas.

\section{TECNOLOGÍAS dE LA INNOVACIÓN Y LA COMUNICACIÓN}

La Consejeria de Educación de la Comunidad de Madrid, siguiendo con su apuesta decidida por el fomento del uso educativo de las Tecnologías de la Información y la Comunicación en los contextos escolares, sigue desarrollando actuaciones entre las que destacar tres por el número de profesores y centros implicados.

1. El Proyecto Modernización y Mejora de los Centros de Educación Secundaria que se viene desarrollando desde 2007, y cuyos datos cuantitativos son actuaciones en los 326 centros de Educación Secundaria de la Comunidad de Madrid mediante la dotación de Portátiles/Tablet PC para uso del profesorado, nuevos ordenadores en Departamentos didácticos, videoproyectores para uso del profesorado en aulas, nuevos puntos de red en espacios sin conectividad actualmente, zonas con cobertura wifi, puntos de red en aulas, y PDA para uso personal de profesores. Todo ello con un presupuesto total cercano a los veintiocho millones de euros.

2. El Plan de Integración de Ordenadores y Pizarras Digitales en Aulas de Educación Infantil y Primaria que ha previsto, para el presente curso escolar 2008-2009, la dotación de ordenadores para las aulas ordinarias y de pizarras digitales interactivas. Este curso participan en el Plan 180 Centros Públicos Bilingües de Educación Infantil y Primaria de la Comunidad de Madrid, 22 Centros Públicos de Educación Especial y 50 centros públicos a propuesta de las Direcciones de Área Territorial, hasta completar las disponibilidades presupuestarias. Se seleccionan preferentemente centros que hasta el momento sólo han contado con recursos TIC basados en aulas informáticas de uso compartido, representando este proyecto la implantación generalizada en todas las aulas de nuevos recursos TIC junto a la dotación de pizarras digitales. Para ello se ha previsto la dotación de ordenadores con instalación de red, pizarras digitales y videoproyectores instalados en techo, tablet pc para centros de educación especial así como conjuntos de mobiliario adaptados al material suministrado.

Este Plan incluye actuaciones de formación, de apoyo y seguimiento, destinadas a centros y profesores para que estas nuevas infraestructuras y recursos se integren con eficacia en la dinámica de los centros y permitan mejorar la consecución de los objetivos educativos que demanda la actual sociedad de la información y la calidad del sistema educativo madrileño. La formación prevista afecta a unos 5.000 maestros a quienes el aprendizaje de conocimientos sobre el manejo del correo electrónico en Educamadrid, de la red del centro y sus posibilidades para compartir recursos, del uso del videoproyector y de la pizarra digital interactiva, de las aplicaciones didácticas del equipamiento recibido y de la elaboración de materiales didácticos les permitirá estar en condiciones de hacer un uso adecuado de los recursos con grandes beneficios para sus alumnos.

3. La Formación en línea propia, que por primera vez es ofertada por la Consejería de Educación, tiene por objeto convertir a Madrid en una región pionera en el uso de esta herramienta. En el primer año de implantación se ofertan 23 cursos en línea diseñados especialmente para atender las demandas de más de 2500 profesores madrileños este curso. Se realizan dos convocatorias durante el curso y los resultados que refleja la primera de ellas son totalmente satisfactorios. Esta formación contribuye a la mejora de las competencias profesionales del profesorado a la vez que potencia el uso de las tecnologías de la información y la comunicación y reduce la movilidad del profesorado. Prueba de la gran adaptación del profesorado de la Comunidad de Madrid es el número de solicitudes que cuadruplica la oferta inicial.

\section{ActividADES EXTRAESCOLARES}

Desde la Consejería de Educación se fomenta la realización de actividades extraescolares fuera del horario lectivo, a través de subvenciones o certámenes de premios. La finalidad de estas actuaciones es la de provocar una mayor identificación entre el estudiante y su centro escolar. El alumno comparte unas experiencias de relación con sus compañeros y profesores distintas a las del período estrictamente lectivo, y se abre a intereses que pueden servirle para la 
vida profesional o para aceptar más fácilmente las normas académicas. Estas actividades mejoran además la disciplina escolar: cualquier actividad, por muy gratificante o creativa que sea, se desarrolla sólo a través de una organización y una jerarquía que todos deben respetar, lo que crea una costumbre que sirve para el resto de la vida en el centro.

Los certámenes más consolidados son el de Coros Escolares y el de Teatro Escolar. Con ellos se crea una incipiente sensibilidad artística que complementa la formación académica y la del individuo en general. La convocatoria de Teatro contribuye además a la mejora de las competencias básicas que promueve la Consejería de Educación, a través de iniciativas como el Plan General de Mejora de las Destrezas Indispensables o el Plan de Fomento de la Lectura. Coincidiendo con la puesta en marcha del programa de Colegios Bilingües de la Comunidad de Madrid y como un modo de estimular el uso de este idioma en edades tempranas se añadió a las bases del certamen la modalidad de teatro en inglés para Primaria.

Además de las convocatorias de premios, la concesión de subvenciones a los centros para que adquieran material destinado a la realización de actividades escénicas, musicales o deportivas se ha revelado como un elemento dinamizador de estas iniciativas. Se pretende ayudar a los colegios e institutos para que la falta de material no sea un obstáculo a cuantas iniciativas deportivas o artísticas quieran promover.

En la misma línea de trabajo, pero con el carácter de proyecto innovador experimental en un número de centros de la Comunidad, se inició hace un año un programa de Cine, llamado Plató 08. Su objetivo es iniciar a los jóvenes en el lenguaje cinematográfico, tanto en su versión clásica como en los formatos digitales más recientes. Además de la formación en cine y de la educación de la mirada, este curso puede tener la utilidad de mostrar a los jóvenes, para su futuro, el amplio campo profesional que abarca la industria cinematográfica.

\section{Campeonatos escolares}

Con el fin de reforzar el valor educativo, social y cultural del deporte, el Parlamento Europeo estableció el año 2004 como "AÑO EUROPEO DE LA EDUCACIÓN A TRAVÉS DEL DEPORTE". Desde ese mismo año la Comunidad de Madrid convocó la Primera Edición de los Campeonatos Escolares de la Comunidad de Madrid.

Estos Campeonatos Escolares son una iniciativa conjunta de la Consejería de Educación y la Consejería de Deportes, cuyo objetivo es fomentar la práctica deportiva entre los jóvenes estudiantes de los Institutos Públicos madrileños. Para ello se cuenta con un equipo de profesionales del mundo de la educación y miembros de las federaciones deportivas madrileñas, que han permitido darle la calidad, el nivel deportivo y educativo necesario, para hacer de ellos uno de los encuentros más importantes y atractivos para los jóvenes estudiantes.

Las modalidades deportivas en las que los alumnos madrileños de entre 12 y 17 años pueden participar son: ajedrez, bádminton, baloncesto, balonmano, fútbol sala, hockey, judo, taekwondo y voleibol.

El Programa tiene entre sus objetivos hacer que el deporte se convierta en un hábito en la vida diaria de los jóvenes, integrar la práctica deportiva en la rutina escolar de los Institutos de Educación Secundaria, fomentar la práctica de los diferentes deportes fuera del horario lectivo entre jóvenes con edades proclives al abandono, incrementar la socialización e integración de los alumnos de los diversos Institutos de Educación Secundaria de la Comunidad, potenciar los valores que definen el juego limpio, facilitar un recurso educativo y formativo potenciando la utilización de las Tecnologías de la Información y de la Comunicación a través del espacio virtual "enredados", ubicado en la página web de Campeonatos Escolares y reformar, acondicionar y mejorar las instalaciones deportivas de estos centros para su utilización en horario de tarde durante el desarrollo de los Campeonatos pero sin duda mejorando los espacios deportivos que disfrutan todos los alumnos matriculados en el centro en las clases de Educación Física.

La trayectoria de estos cinco años ha supuesto el aumento de la participación de los IES de 155 a 275 en la actualidad, lo que supone prácticamente el $90 \%$ de los institutos de la Comunidad de Madrid, un aumento del número de alumnos que hoy practican deporte en su tiempo de ocio. Los 15.000 alumnos de la primera edición se han convertido en más de 28.000 en la quinta; la participación femenina ha supuesto un éxito si se analiza el incremento de chicas en las distintas modalidades a lo largo de las cinco ediciones, 
pasando de las 5.000 inscritas en la primera edición a las casi 10.000 en esta última; el Juego Limpio ha tomado protagonismo en estos Campeonatos desde el principio lo que marca diferencia con otras competiciones, el espacio "enredados" se pone en marcha en la III Edición y en esta $\checkmark$ se intenta potenciar la participación de profesores que sin estar implicados directamente en el Programa pudieran utilizar las TIC y la actividad físico-deportiva como elemento motivador en sus clases y programación; la mejora de instalaciones y equipamientos deportivos ha sido valorado muy positivamente por toda la comunidad educativa (alumnos, profesores y familias).

\section{+ Educación}

El deseo de innovación acompaña siempre al de mejora y éste también se refleja en la imagen y el los servicios que, a través de Internet la comunidad ofrece a profesores, alumnos y padres. El diseño, dentro de Madrid.org, de un portal de educación moderno, ágil, accesible y completo permite mejorar la comunicación y la información sobre todo cuanto acontece en el mundo educativo. La comunidad educativa dispone de una herramienta útil que integra en un solo portal los principales subportales de la Consejería como Educamadrid, Mi+D, Emes, Personal, así como las Direcciones de Área Territorial. Este esfuerzo de modernización permite a los usuarios una navegación amigable y un acceso rápido a la información requerida.

\section{FORMACIÓN DEL PROFESORADO}

La formación del profesorado también se ha visto mejorada mediante una nueva redefinición tanto de una red de formación que había quedado obsoleta como de un sistema que mantenía el desarrollo de modelos agotados. Ambos elementos precisaban de una restructuración con el fin de actualizar y adaptar el sistema de formación en su conjunto a las nuevas necesidades y a las nuevas demandas de los usuarios. La comunidad de Madrid cuenta en estos momentos con un modelo de formación del profesorado renovado, moderno, dirigido de manera muy específica a la mejora de las competencias profesionales de los profesores y por lo tanto con incremento del impacto de las mismas en los alumnos, objetivo principal y final del sistema educativo.

Este ámbito, clave para un correcto funcionamiento de la educación, y que se ve reforzado por la formación en línea mencionada anteriormente, debe ser objeto permanente de la innovación.

\section{Pruebas finales}

Innovadoras y pioneras son las pruebas que desde hace unos años la Consejería de Educación realiza en el último curso de primaria y desde este año también en tercero de la ESO. Estas pruebas Ilamadas de conocimientos y destrezas indispensables producen un efecto positivo en el sistema. Por un lado permite que padres, profesores y alumnos estén informados sobre la evolución de los aprendizajes de sus hijos al tiempo que permite, mediante la aplicación de pruebas basadas en unos estándares, conocer el grado de adquisición de los conocimientos indispensables de nuestros jóvenes. Por otro generan una reacción en los centros que conduce al refuerzo de unos objetivos a los que en ocasiones no se daba la necesaria importancia. Además, introduce en todos los estamentos de la comunidad educativa la cultura de la evaluación que tanto precisa nuestro sistema educativo, ya que éste se caracteriza, a diferencia de la mayoría de los países con los que nos comparamos, por la ausencia de controles y de evaluaciones.

\section{Publicaciones}

Desde la red de formación del profesorado, cuyos centros se denominan "Centros de innovación y formación" surgió la necesidad de recoger y difundir aspectos relacionados con ambos temas. La revista "Innovación y formación" vio la luz en la primavera de 2007 con el objetivo de informar a los profesores sobre los temas de innovación que más interés pudieran tener para ellos. Así, junto a un variado contenido relacionado con diferentes aspectos educativos, cada número incorpora un dosier sobre un tema innovador de relevancia. La pizarra digital, el Portfolio europeo de las lenguas o los planes de mejora educativa han sido hasta 
el momento los temas tratados. Numerosas aportaciones de los propios docentes enriquecen el intercambio y la difusión de experiencias exitosas realizadas en los centros educativos.

\section{LA INNOVACIÓN EDUCATIVA en la Comunidad de Madrid}

Innovación es sinónimo de mejora, de avance, de progreso y en consecuencia debe formar parte de la hoja de ruta de cualquier administración educativa. La enumeración y breve descripción de diversos elementos relacionados con la innovación educativa dan una idea de la importancia que la Consejería de Educación de la Comunidad de Madrid concede a la misma.

La innovación no se justifica mediante el desarrollo de acciones aisladas, sino de todo un conjunto de medidas que crean sinergias entre sí. El objetivo es bien claro: disponer de un sistema educativo lo más moderno y eficaz posible que permita ofrecer a los jóvenes madrileños la mejor formación posible y las mayores oportunidades para que puedan competir al menos en igualdad de condiciones con los jóvenes de Europa y del mundo entero.

El futuro de nuestros jóvenes está en nuestras manos y debemos esforzarnos por conseguir que ese futuro sea el mejor posible.

Recibido: 16 de marzo de 2009

Aceptado: 16 de abril de 2009 